\title{
La vraie vie est ailleurs... fonction métonymique des réseaux dans quelques publicités britanniques pour le téléphone
}

\section{Marie-Hélène Fries-Verdeil}

\section{(2) OpenEdition}

Journals

Édition électronique

URL : http://journals.openedition.org/asp/2390

DOI : 10.4000/asp.2390

ISBN : 978-2-8218-0380-0

ISSN : 2108-6354

\section{Éditeur}

Groupe d'étude et de recherche en anglais de spécialité

\section{Édition imprimée}

Date de publication : 1 décembre 1999

Pagination : 165-176

ISSN : 1246-8185

Référence électronique

Marie-Hélène Fries-Verdeil, «La vraie vie est ailleurs... fonction métonymique des réseaux dans quelques publicités britanniques pour le téléphone », ASp [En ligne], 23-26 | 1999, mis en ligne le 21 juin 2011, consulté le 02 mai 2019. URL : http://journals.openedition.org/asp/2390 ; DOI : 10.4000/ asp. 2390

Ce document a été généré automatiquement le 2 mai 2019.

Tous droits réservés 


\title{
La vraie vie est ailleurs... fonction métonymique des réseaux dans quelques publicités britanniques pour le téléphone
}

\author{
Marie-Hélène Fries-Verdeil
}

\section{Introduction}

1 Il est difficile de se promener dans la rue aujourd'hui, de part et d'autre de la Manche, sans rencontrer au moins un utilisateur de téléphone portable. La radiotéléphonie connaît en effet, depuis le début des années 90, une expansion spectaculaire. Et ce développement des réseaux de portables est accompagné de campagnes publicitaires nombreuses et bien orchestrées, que ce soit sur les murs de nos villes, ou au petit écran. À la télévision, les publicités pour les téléphones mobiles jouent essentiellement sur trois registres: l'innovation technologique, la baisse des coûts tarifaires, et les relations amoureuses, amicales ou familiales que le téléphone permet d'entretenir. Ces messages publicitaires renvoient à leur tour à trois types de réseaux différents: les réseaux physiques de télécommunications (qui ont mis la radiotéléphonie à la portée du grand public), les réseaux commerciaux qui les accompagnent (et qui commanditent les campagnes de publicité), et enfin tous les réseaux sociaux qui utilisent le portable pour leur survie ou leur développement, et motivent donc l'extension de la radiotéléphonie.

2 À partir d'une réflexion préliminaire sur le rôle respectif des métaphores et des métonymies dans le concept de réseau, je vais ensuite partir de l'hypothèse selon laquelle tout réseau de communication fonctionnerait de façon métonymique pour analyser différents niveaux de métonymies, dans trois publicités britanniques.

3 En français comme en anglais, le concept de réseau, ou de "network» est issu d'une métaphore textile, puisque «net », tout comme le vieux mot français rets, signifie le filet. Michel Serres, par exemple définit un diagramme en réseau comme : 
une pluralité de points (sommets) reliés entre eux par une pluralité de ramifications (chemins) (1968:11) expérimental (physique, par exemple), soit théorique (politique, mathématique, etc.). Dans le cas des portables qui nous intéressent ici, on peut distinguer trois sortes de réseaux : les réseaux physiques de radiotéléphonie, les réseaux commerciaux permettant de développer ce nouvel usage du téléphone, et les réseaux sociaux qui utilisent les «télécom» pour leur fonctionnement propre. Dans tous ces cas, les points peuvent représenter des êtres humains (tenant à la main un portable, détenant un abonnement de radiotéléphonie, ou maintenant des liens d'ordre familial ou amical), mais ils peuvent aussi désigner des objets inanimés servant de médiation entre deux abonnés (relais de télécommunications, formules d'abonnement, points communs d'ordre idéologique). réseau une représentation visuelle ${ }^{1}$. En revanche, dès qu'on cherche à étudier le fonctionnement d'un réseau dans le temps, à voir comment l'information se déplace d'un point à l'autre, on entre dans le champ de la métonymie, d'un déplacement du sens par le biais d'une relation. Il ne peut y avoir réseau que si les chemins entre les points sont parcourus, si les connexions servent. Et Michel Serres, dans sa définition d'un diagramme en réseau, prend soin d'ajouter :

il représente un état quelconque d'une situation mobile. (1968:11)

6 Dans quelle mesure cette analyse s'applique-t-elle aux réseaux de portables? On peut remarquer tout d'abord que le téléphone est un média qui véhicule du sens par l'intermédiaire de sons. Et ce déplacement se fait «en temps réel » puisque le temps de parcours du son entre un poste émetteur et un poste récepteur est infime, même pour des distances de plusieurs milliers de kilomètres. Téléphoner, c'est donc être à la fois ici et ailleurs, ici en personne, et ailleurs par la voix. Ce caractère d'ubiquité partielle, ajouté à la mobilité des portables, explique la fluidité des réseaux de radiotéléphonie, caractérisés par un déplacement constant des points, comme des chemins. Dans ce maillage en perpétuelle évolution, la seule constante est la permanence d'un déplacement simultané de sons et de sens, donc de métonymies.

7 En partant de l'hypothèse que tout réseau de communication fonctionne potentiellement de façon métonymique, j'essayerai maintenant d'analyser les différentes utilisations du concept de réseau dans des publicités britanniques sur le téléphone portable, datant de juin $1998^{2}$. Ces campagnes publicitaires se situent dans un contexte de développement spectaculaire. Les mobiles représentaient presque $10 \%$ du marché mondial des télécommunications en 1996, contre un peu plus de $3 \%$ seulement en 1990. Le RoyaumeUni occupe une place enviable dans ce contexte global favorable, puisque plus de $16 \%$ de ses recettes téléphoniques provenaient des portables en 1996, ce qui plaçait les Britanniques au deuxième rang mondial, derrière le Japon (cf. annexe 1). La dérégulation du marché britannique des télécommunications a pu contribuer pour partie à ce succès. Elle a en tout cas remplacé le monopole de British Telecom par une situation de concurrence entre plusieurs réseaux rivaux. Et l'on comprend aisément dans ces conditions l'intérêt de campagnes publicitaires, pour attirer l'utilisateur potentiel. Le corpus que j'ai étudié contenait des publicités pour deux firmes nordiques, Ericsson et Nokia, et des opérateurs britanniques, Cellnet, Orange, et Vodafone ${ }^{3}$. Les argumentaires mis en scène s'appuyaient sur trois logiques : une description de la nouveauté technologique que constitue la radiotéléphonie, une annonce de rabais sur le coût des communications, 
ou une évocation des liens sociaux que le téléphone peut contribuer à vivifier. Ils mettaient donc l'accent tour à tour sur le réseau physique de radiotélécommunications, le réseau commercial qui l'accompagne, ou le réseau social qui le sous-tend.

\section{Publicité pour Orange}

8 La publicité pour la firme Orange illustre la première option, en vulgarisant la technique du radiotéléphone. L'onde transportant les mots d'une conversation téléphonique d'un poste émetteur à un poste récepteur est représentée physiquement, au cours d'un petit dessin animé au graphisme naï. On entend un monsieur âgé, assis dans un fauteuil, proférer des paroles incompréhensibles, qui se matérialisent immédiatement sous forme de lettres inscrites sur une vague de couleur orange. Le choix de cette couleur est bien sûr guidé par le nom de l'annonceur, et la vague représente l'onde radio qui véhicule les sons de la conversation, et remplace, pour les téléphones portables, les lignes ou câbles de nos téléphones fixes. Dans son trajet jusqu'à la vieille dame qui reçoit cette conversation téléphonique en tricotant dans son fauteuil, l'onde rencontre certains obstacles (le mur de la maison de départ, un autobus, un marcheur qui promène son chien), en évite d'autres (les usines), et parcourt de vastes espaces libres (les bois, les pâturages à moutons). Ces éléments ont été choisis avec soin pour rappeler des révolutions passées (le pré ouvert fait penser aux "enclosures", et l'architecture surannée de l'usine à la révolution industrielle), tout en décrivant l'évolution présente. Dans son trajet, l'onde traverse en effet les principaux types de paysages ruraux et urbains du Royaume-Uni, illustrant par là le fait que le réseau Orange couvre la quasi-totalité du territoire britannique. Et la voix off fait écho à cette interprétation :

Orange believes that everyone should always be able to get through. That's why we have installed more transmitters, in more places, that any other digital network. (cf. annexe 2)

Dans ce contexte de nouveauté technique, de dérégulation économique, et de compétition entre différents réseaux de téléphones portables, des arguments «objectifs» tels que le taux de couverture du territoire et la qualité de la transmission sont porteurs. À quoi servirait un portable, si l'émetteur téléphonique restait muet lorsqu'on désirait s'en servir, ou si l'interlocuteur était inaudible? On retrouve ici l'argumentaire traditionnel des publicités scientifiques, informer pour mieux persuader ${ }^{4}$. Mais l'originalité consiste ici à visualiser l'ubiquité propre au téléphone, puisque, grâce à son portable, le monsieur âgé peut se retrouver présent à la fois chez lui (en personne), et auprès de la vieille dame (par ses paroles et ses rires). La réaction de son interlocutrice, qui ne tient pas même l'ombre d'un téléphone à la main, et se met à sourire en continuant tranquillement son tricot, comme si elle le voyait en face d'elle, rend visible cette ubiquité. La mise en scène du téléphone portable, dans le petit dessin animé pour la firme Orange, rend donc visibles à la fois le réseau de radiotéléphonie (qui permet la transmission de sons), et le réseau social liant les deux interlocuteurs (qui opèrent la transformation des sons en conversation). La métonymie apparaît alors comme constitutive de l'expérience téléphonique, puisqu'il ne peut y avoir communication sans déplacement de sens. 


\section{Publicité pour Cellnet}

10 Néanmoins pour nombre d'utilisateurs de portable, les prouesses de réalisation technique de ce média importent moins que le coût des conversations, et la guerre des tarifs joue un rôle important dans la compétition entre les différents opérateurs téléphoniques. On voit alors que le réseau physique constitué par les téléphones portables et certaines fréquences d'ondes radio se double d'un réseau commercial composé d'un opérateur, de ses abonnés, et des différents types de tarifications proposés. Le partenaire de British Telecom spécialisé dans les téléphones mobiles, Cellnet, a choisi cet angle d'attaque pour faire connaître sa formule « First for Families ». Le cadre de cette publicité est un paysage de vacances. La caméra balaye une prairie au vert typiquement britannique, avant de plonger sur un pittoresque village de bord de mer où un jeune footballeur est en train d'utiliser son portable, un ballon à la main (allusion à la coupe du monde de football, qui avait lieu à ce moment-là). Pendant ce temps, une voix off présente la formule « First for Families ", en décrivant le principe de la réduction (cf. annexe 3):

Now your family can have up to six Cellnet mobile phones, each with ten numbers...

avant de développer ses conséquences, en termes d'économies sur les factures de téléphone :

up to $50 \%$ extra discounts

12 L'expression «First for Families » s'impose de prime abord à l'esprit du public-cible comme un clin d'œil parodique à la formule d'abonnement «Friends and Families» de British Telecom, dont elle reprend la structure rythmique, et l'allitération en $\mathrm{f}$ :

It's the same great idea for mobile phones as BT's "Friends and Family" is for homes

Mais, alors que «Friends and Families» orientait le message publicitaire vers la seule sociabilité, «First for Families » joue sur deux registres différents. On peut l'entendre en effet comme « le $n^{\circ} 1$ (des réseaux de téléphones portables) pour les familles », ou comme «d'abord (en priorité) destiné aux familles ». La première interprétation constitue une hyperbole, que la voix off développe ensuite dans son argumentaire ("great idea »), et utilise pour sa démonstration. Les possibilités offertes par la formule d'abonnement «First for Families » sont en effet d'abord présentées de façon neutre, puis reformulées de façon à gonfler les chiffres. Ainsi, le nombre de numéros de téléphone bénéficiant d'une réduction spéciale est-il d'abord annoncé sous la forme :

up to six cellnet mobile phones each with ten numbers...

14 avant que la multiplication ne soit effectuée : «60». De la même façon, la réduction consentie est présentée par rapport au chiffre maximum (« up to 50\% »). Ce raisonnement conduit à une dissymétrie calculée: $50 \%$ de réduction pour 60 numéros différents. À première vue, l'ampleur de la baisse de tarif semble presque compenser la multiplication de communications téléphoniques potentielles. En fait, il n'en est rien, puisque le nombre de téléphones est multiplié par six, alors que le tarif des communications n'est divisé que par deux.

15 Les chiffres 60 et 50 ont donc ici une valeur rhétorique plus que mathématique ${ }^{5}$, et servent à conforter la prétention affichée par la firme Cellnet d'offrir aux familles un service plus souple et meilleur marché que leurs concurrents. Dans un marché britannique qui a vu le nombre et la durée des communications par ligne principale augmenter entre 1990 et 1996, alors que dans le même temps la recette téléphonique 
moyenne par communication diminuait de presque $5 \%$, le message est porteur (cf. annexe 1). La première interprétation de "First for Families" (numéro un pour les familles) joue donc sur un glissement métonymique du réseau physique de télécommunications (signifié par la présence discrète d'un portable) au réseau de commercialisation de Cellnet. L'important ici n'est pas de savoir comment marche un portable, mais combien il coûte. Dans la deuxième interprétation de «First for Families » (en priorité destiné aux familles), en revanche, l'accent est mis sur les avantages pratiques de cette formule dans la vie quotidienne: six téléphones («up to six Cellnet mobile phones ») pour un seul abonnement. L'image de l'adolescent utilisant son portable dans un idyllique paysage de vacances exprime à la fois la liberté (on peut téléphoner de n'importe où, ou presque, avec un portable), et le plaisir de communiquer (suggéré également par le ballon de football). La fin (la communication) justifie ici les moyens (les télécom), et le développement du réseau de radiotéléphonie se trouve motivé, dans un deuxième glissement métonymique, par les besoins de réseaux familiaux ou amicaux qui lui ont préexisté.

\section{Publicité pour Vodafone}

La présence du couple âgé et de l'adolescent dans les publicités pour Orange et Cellnet montre bien qu'au-delà de l'innovation technologique et des contraintes économiques, les publicitaires se doivent aussi de prendre en compte les motivations des abonnés. Qu'estce qui pousse un utilisateur à décrocher son téléphone ? Pour le grand public, qui est la cible des publicités télévisées, il s'agit trois fois sur quatre de joindre famille et amis ${ }^{6}$. Les réseaux physiques de télécommunications doivent donc leur extension à des réseaux sociaux qui leur préexistent, et qu'ils aident à subsister malgré un éclatement géographique de plus en plus fréquent, puisque l'ubiquité téléphonique permet de pallier en partie la distance physique ${ }^{7}$.

L'entreprise Vodafone a clairement mis en valeur ce glissement métonymique de la connexion physique au lien social dans sa publicité pour les conversations au tarif de deux pence par minute. Pas ombre ici de portable, ni même de conversation téléphonique. Trois scènes muettes se succèdent. On voit d'abord un témoin à un banquet de mariage, qui s'apprête à commencer son discours, debout, un texte à la main. Puis se succèdent un papa parlant à son fils et le faisant sauter dans ses bras sur un banc public, et enfin une discussion amicale et animée dans un «pub». Simultanément, deux lettres $p$ se superposent à chaque scène, en rouge sur l'écran, avant d'être complétées par les expressions : "prepared words", "proper words» et "popular words", chacun des adjectifs « prepared », « proper », et « popular » contenant les deux p. La voix off annonce ensuite l'offre promotionnelle de Vodafone : deux pence la minute.

Ce jeu sur le doublement de la lettre p s'appuie sur une antanaclase ${ }^{8}$ (c'est-à-dire une figure de rhétorique où un même mot est utilisé avec deux sens différents). Au cours de la publicité, les deux lettres $\mathrm{p}$ sont en effet complétées par des caractères en noir pour former les mots "prepared", "proper", et "popular». Mais, en même temps, leur couleur rouge qui tranche sur le noir et blanc des images attire l'attention des téléspectateurs, et les prépare à la révélation finale : « $2 p$ a minute », où le mot " pence » est familièrement abrégé sous la forme « $p$ ». Le caractère d'imprimerie $p$ renvoie donc ici à la fois à une lettre, et à une monnaie, et crée un effet de surprise, sur lequel est construite la publicité pour Vodafone. Cet effet de surprise est renforcé par l'image. Les 
trois scènes choisies représentent en effet des situations typiques de communication en face à face. On a de la peine à imaginer, par exemple, de longues conversations téléphoniques avec un bébé. Inversement, malgré l'absence de sons, les images sont parlantes, et la charge d'émotion y est forte, qu'elle se traduise par des exclamations au pub, s'exprime par les mimiques père-fils, ou se trahisse par les tremblements de la cravate du témoin. Mais tous ces obstacles à la communication à distance sont magiquement levés par la voix off :

Vodafone new local off-peak call rates for both evenings and weekends? Just $2 \mathrm{p}$ a

minute. (cf. annexe 4)

Sous entendu: dans ces conditions, le prix n'est plus un obstacle à la longueur des communications, ce qui donne aux émotions le temps de s'exprimer, et permet de vivre par téléphone des situations de communication qui nécessitaient jusque-là la présence physique d'un interlocuteur. Ce message est bien adapté au marché téléphonique du Royaume-Uni, où le taux de couverture est de $109 \%$ (ce qui signifie qu'on compte 109 lignes téléphoniques pour 100 foyers) ${ }^{9}$. Si le marché des lignes principales est saturé, il reste à diversifier les usages du téléphone, en mettant en lumière de nouveaux besoins.

Cette ouverture à de nouvelles situations de communication va de pair avec la visée d'un nouveau public-cible: les messieurs. Le fait d'avoir placé des acteurs, plutôt que des actrices, dans les trois scènes de la publicité est significatif : les hommes ont en effet la réputation d'être moins à l'aise que les femmes dans ce qu'ils ont baptisé le «bavardage téléphonique ", c'est-à-dire dans des conversations orientées vers la sociabilité plutôt que vers la rentabilité. Deux sociologues français, Curien et Périn (1983), ont par exemple mis en relief un partage des tâches dans les relations entre une famille et son environnement. Les hommes prendraient plus volontiers en charge les relations d'ordre administratif ou économique, alors que les femmes s'orienteraient davantage vers les contacts privés. Dans cette perspective, les hommes constituent un public-cible de choix, qui va pouvoir découvrir le plaisir de cultiver des relations familiales ou amicales dans son temps libre, grâce aux tarifs réduits consentis en soirée, et le week-end.

21 Dans la publicité pour Vodafone, le coût d'une communication est donc justifié par son contenu, et par les relations qu'elle permet d'entretenir. Ici, l'existence d'un réseau physique de radiotéléphonie est tenue pour acquise et le déplacement métonymique se fait directement du réseau économique au réseau social. L'antinomie entre la modicité des tarifs ( $2 \mathrm{p}$, les deux lettres $\mathrm{p}$ qui apparaissent sur l'écran), et l'ampleur des enjeux communicationnels («prepared words, proper words, popular words») développe progressivement cette métonymie que le slogan final résume en quatre mots : « the word is Vodafone ». On assiste ici à un glissement de la fin («the word», la parole) aux moyens (le réseau de télécommunication Vodafone). Cette métonymie se double avec le mot "word" d'une synecdoque, c'est-à-dire d'une figure de style où la partie représente le tout. "Word» signifie en effet à la fois un mot en particulier (en écho à "prepared words, proper words, popular words ») et tous les mots, la parole, le logos grec. Cette synecdoque appelle à son tour deux références culturelles fort différentes. On peut y lire d'une part une allusion parodique à l'expression familière «mum's the word", prise ici à contre-pied et, d'autre part, un écho solennel au prologue de l'évangile de Jean :

In the beginning was the Word, and the Word was with God, and the Word was God.

(Jean $1: 1$, cité dans la version du roi Jacques) sémantique à un autre) ${ }^{10}$. S'il y a équivalence, au début de l'évangile de Jean, entre logos 
et theos, c'est parce que Dieu partage avec sa Parole le même pouvoir créateur. La puissance apparaît donc ici comme le caractère commun liant métaphorisant et métaphorisé. Qu'on remplace Dieu par Vodafone, l'attribut commun de puissance subsiste, puisque le logos demeure Parole créatrice des mondes, donc porteuse d'un pouvoir de communication qui s'inscrit dans le réel. Par le jeu d'une synecdoque et d'une métaphore, le slogan «The word is Vodafone » recèle donc un double glissement métonymique, de la technologie au commercial et au social. Il peut en effet s'entendre de deux façons : soit "Le mot (à retenir) c'est Vodafone" (le réseau commercial), soit "La Parole (la communication, le réseau social), c'est Vodafone (le réseau de télécommunications) ».

\section{Conclusion}

En conclusion, les trois publicités pour Orange, Cellnet, et Vodafone illustrent bien l'hypothèse selon laquelle tout réseau de communication fonctionne de façon métonymique. La métonymie apparait comme constitutive de l'expérience téléphonique avec Orange, elle opère un glissement de l'innovation technologique aux coûts avec Cellnet , et des tarifs téléphoniques au besoin de communiquer avec Vodafone. Ces différents transferts de sens sont rendus possibles par l'ubiquité téléphonique, qui crée un pouvoir, tout en creusant un mal-être. Être détenteur d'un portable confère certes le pouvoir d'être ici et ailleurs à chaque instant, mais les limites de nos journées et de nos budgets nous empêchent de multiplier ces ailleurs à la mesure de notre désir. La publicité gomme subtilement l'expérience de nos limites et attise notre désir d'ubiquité en nous persuadant que la vraie vie est ailleurs, et que pour y avoir pleinement accès il faut simplement être branché... au moindre coût.

Cet article n'aurait pas vu le jour sans l'amabilité de Sally Gauthier, et de Jim Hutchinson qui a pris la peine d'enregistrer les publicités étudiées. Les suggestions faites par Sonia Lacabanne, Evelyn Perry et Jonathan Upjohn lors du colloque du GERAS 1999 m'ont été fort utiles pour explorer la richesse polysémique des slogans publicitaires.

\section{BIBLIOGRAPHIE}

Bornot, Françoise et Anne Cordesse. 1981. Le téléphone dans tous ses états. Le Paradou : Actes Sud.

Cameron, Lynne. 1999. « Operationalizing "metaphor" for applied linguistic research ». In Cameron, Lynne \& Graham Low (dir.), Researching and Applying Metaphor. Cambridge : Cambridge University Press.

Curien N. et P. Périn. 1983. « La communication des ménages, une cartographie socioéconomique ». Réseaux 2, 35-59.

Deignan, Alice. 1999. « Corpus-based research into metaphor ». In Cameron, Lynne \& Graham Low (dir.), Researching and Applying Metaphor. Cambridge : Cambridge University Press. 
Flichy, Patrice. 1997. « Perspectives pour une sociologie du téléphone ». Réseaux (communicationtechnologie-société) 82-83, 7-19. <http://www.persee.fr/web/revues/home/prescript/article/ reso_0751-7971_1997_num_15_82_3055>

Fries, Marie-Hélène. 1993. «Contraintes descriptives et libertés rhétoriques dans la publicité à caractère scientifique ou technique ». ASp 3, 355-370.

Fries, Marie-Hélène. 1998. « Les nombres dans la publicité scientifique ou technique : quand la langue de l'industrie devient l'industrie de la langue ». ASp 19-22, 121-134.

Serres, Michel. 1968. Hermès, ou la communication. Paris : les Éditions de Minuit.

Suhamy, Henri. 1997 [1981]. Les figures de style. $8^{\mathrm{e}}$ éd. coll. Que sais-je ?. Paris : Presses universitaires de France.

Verlinde, Thierry. 1997. « Le point sur... les usages du téléphone dans le monde ». Réseaux (communication-technologie-société) 82-83, 269-282.<http://www.persee.fr/web/revues/home/ prescript/article/reso_0751-7971_1997_num_15_82_3071>

\section{ANNEXES}

\section{Annexe 1 - La Radiotéléphonie en chiffres}

\section{Réseaux de télécommunications :}

Les mobiles représentaient 3,5\% du marché mondial des télécommunications en 1990, 9,5\% en 1996 (ce qui correspond à une croissance annuelle de 22,6\%).

Pourcentages des recettes de télécommunications dues aux radiotéléphones en 1996 (source : Verlinde 1997 : 271)

Japon : $22 \%$

Royaume-Uni : $16,2 \%$

États unis : $12,7 \%$

Allemagne : $12,6 \%$

Pays-Bas : $10,6 \%$

France : $7,7 \%$

Espagne : $3 \%$

Réseaux commerciaux au Royaume-Uni (source : Verlinde 1997 : 271)

\begin{tabular}{|l|c|c|}
\hline & $\mathbf{1 9 9 0}$ & 1996 \\
\hline Durée des communications & $2^{\prime} 94^{\prime \prime}$ & $3^{\prime} 08^{\prime \prime}$ \\
\hline Nombre de com. par ligne principale & 1217 & 1225 \\
\hline Recette téléph. moyenne par communication & 59 & $44(-4,9 \%)$ \\
\hline
\end{tabular}

\section{Réseaux sociaux :}

Destinataires des communications privées (France et États-Unis) -(source : Flichy 1997 : 11) 
$40 \%$ famille

$36 \%$ amis et relations

$24 \%$ autres

Annexe 2 - Orange

\begin{tabular}{|l|l|}
\hline Cartoon & off Voice \\
\hline $\begin{array}{l}\text { Dominating orange colours. Full shot on a man sitting in an armchair under } \\
\text { a lamp, with a white garden chair in the background. The man is uttering } \\
\text { incomprehensible words, which materialise in the shape of white letters in } \\
\text { the middle of a waving orange band. The camera then tracks in and follows } \\
\text { the orange wave through the brick walls of the man's house, a double- } \\
\text { decker bus, an ornate iron gate, a parson walking his two dogs, an orchard } \\
\text { with a flock of birds taking flight, a meadow with grazing sheep, and finally } \\
\text { able to get } \\
\text { a fluttering crow. Full shot on an old lady knitting in an armchair in a } \\
\text { thatched cottage, and saying "Yes, dear." The cartoon is reframed into the } \\
\text { logo of the firm, an orange square, with orange written at the bottom. Their } \\
\text { phone number, } 0888 \text { 80 10 80, is written underneath in big type, and their } \\
\text { internet address, www.orange.co.uk, in small characters. }\end{array}$ & $\begin{array}{l}\text { are installing } \\
\text { more } \\
\text { transmitters, in } \\
\text { more places, than } \\
\text { any other digital } \\
\text { network. }\end{array}$ \\
& $\begin{array}{l}\text { To find out more } \\
\text { about the } \\
\text { network that } \\
\text { performs better, } \\
\text { call 0 800 80 10 } \\
80 "\end{array}$ \\
\end{tabular}

Annexe 3 - Cellnet

Pictures

Off Voice 
Extreme long shot on a star-studded sky, with "Another Money Saving First from Cellnet" in big characters in the fore-ground. Dissolves. Extreme long shot on green pastures. The camera tilts down. Low-angle shot on a sea-side village. Full shot on a football player sitting on a wall, holding a ball on his knee, and phoning at the same time. "First for Families" appears in big type on the screen, then dissolves, replaced by " $10 \%$ peak discounts. $50 \%$ offpeak discounts".

The camera tilts further down to the sea and the bottom of the village, then up toward the houses at the top of the village. This high-angle shot is accompanied by the conclusion "Discounts on up to 60 numbers." The camera zooms backward to the green meadows behind, and finally the sky, which darkens to a deep night blue. The sky lights up with the phone number of the firm, 08004050 30, and five purple dots expand into "Cellnet First" and its logo, a white star surrounded by a purple halo.
"Here's more good news from Cellnet 'First for Families'. It's the same great savings idea for mobile phones as BT's 'Friends and Family' is for homes.

Now your family can have up to six Cellnet mobile phones, each with 10 numbers with extradiscounts on every call to those numbers.

That's up to 60 numbers per family, earning up to $50 \%$ extra discounts.

First for Families from Cellnet. Call us. We have a lot to talk about".

\section{Annexe 4 - Vodafone}


Close-up on a best man standing up in front of a wedding-cake. Mediumclose shot of the young man looking nervously at his audience, holding the notes for his speech in his hand. Quote marks and two "p" letters appear in red on the screen. Medium shot on a fearsome old lady dressed up in blue, listening. The phrase "prepared words" fills in the quote, the other letters appearing in white. Medium close shot on the young man speaking, with a blurred back-ground. Focus on the back-ground, with the bride and bridegroom listening.

Close up on a dark-haired man holding a baby near his face and talking to him. Quote marks and two $p$ letters appear in red on the screen. Close up on the baby. The phrase "proper words" fills in the quote, the other letters appearing in white. Extreme long shot on the man, sitting with his baby on a park bench overlooking a big city.

Blurred picture coming into focus. Medium shot on people talking in a pub, with beer on draught in the fore-ground. Quote marks and two "p" letters appear in red on the screen. Close up on a beer glass, then on a white man listening, then a black man speaking. The phrase "popular words" fills in the quote, the other letters appearing in white. Close shot on a young man drawing a circle with his hand, laughing and talking to a young woman.

" $2 p$ a minute" appear in red on the screen against a black backdrop, featuring "2p" in big type, and "a minute" in small letters. Dissolves. The phrase "The word is" appears in white type at the top of the screen, and two glittering red rings, representing the logo of the firm, at the bottom. The white letters dissolve, the two rings become part of the red word Vodafone, topped by a red logo.
"Can you guess how much Vodafone new local off-peak call rate is for both evenings and week-ends?

Just $2 \mathrm{p}$ a minute. For cheaper local off-peak calls, the word is Vodafone."

\section{NOTES}

1. Pour une discussion plus approfondie sur les relations entre les sens non métaphoriques et métaphoriques d'un même mot, voir Deignan 1999 : 177-199.

2. Ces publicités ont toutes été enregistrées sur la chaîne ITV, au moment de la coupe du monde de football 1998.

3. Seuls les opérateurs britanniques seront étudiés ici

4. Pour un développement de cette hypothèse, on peut se reporter à Fries 1993 : 355-370.

5. Sur l'utilisation rhétorique des chiffres dans la publicité, on peut voir aussi Fries 1998 : 121-134.

6. Ce sont en tous cas les chiffres disponibles pour la France et les États-Unis : $40 \%$ famille, $36 \%$ amis, que je me suis permise d'extrapoler au Royaume-Uni, vu leur relative stabilité (cf. annexe 1).

7. En d'autres termes, la fonction principale du téléphone est de pérenniser des relations, plutôt que de les créer. C'est la conclusion à laquelle sont arrivées Françoise Bornot et Anne Cordesse, au terme de leur étude sur le développement du téléphone et la dispersion des communautés traditionnelles: "S'il ne parvient pas lui-même, sinon dans l'exceptionnel, à susciter des relations qui n'existaient pas, [...] le téléphone exerce en revanche pleinement son rôle de 'communication' et devient une dimension majeure de la maintenance des groupes (familiaux ou amicaux) lorsque la dispersion la menace. » (1981 cité dans Flichy : 1997 : 8) 
8. L'antanaclase est la figure qui sous-tend le plus souvent les jeux de mots. Henry Suhamy la définit de la façon suivante: "L'antanaclase consiste à reprendre un mot dans une phrase en opposant deux sens différents qu'il peut assumer » (1997: 62)

9. cf. Verlinde $1997: 274$

10. Cette définition très générale de la métaphore a le mérite d'être opérationnelle ici. Pour une discussion plus approfondie au sujet de la définition des métaphores en linguistique appliquée, on peut se référer à L. Cameron 1999 : 3-28.

\section{RÉSUMÉS}

Le but de cet article est d'analyser le rôle des métonymies dans les publicités britanniques sur le téléphone portable. L'hypothèse selon laquelle les réseaux de radiotéléphonie fonctionneraient de façon métonymique plutôt que métaphorique, bien que le mot réseau soit issu d'une métaphore textile, est appliquée à trois publicités britanniques sur le téléphone portable, datant de juin 1998. Avec la firme Orange, la métonymie apparaît comme constitutive de l'expérience téléphonique. La filiale de British Telecom spécialisée dans les téléphones mobiles, Cellnet, a choisi de jouer sur les tarifs, et d'énumérer toutes les économies que sa formule «First for Families » rend possible. La communication est alors métonymiquement représentée par son coût. L'entreprise Vodafone a clairement mis en valeur un glissement métonymique de la connexion physique au lien social dans sa publicité pour les conversations au tarif de deux pence par minute en faisant se succéder trois scènes riches, sans y inclure l'ombre d'un téléphone. On voit donc comment les publicités pour le téléphone portable fondent leur argumentaire sur différents niveaux de métonymies, du réseau de télécommunications aux réseaux sociaux, en passant par des réseaux économiques.

This article analyses the use of metonymy in British advertisements on mobile phones. Starting from the assumption that the rhetoric of telephone networks is based on metonymy rather than metaphor, in spite of the metaphorical etymology of the word «network», three advertisements from June 1998 are analysed. The firm Orange has chosen to materialise the wave carrying the words of a phone talk from speaker to listener via a humorous cartoon, stressing the inherent metonymic character of the telephone experience. The subsidiary of British Telecom specialising in mobile phones, Cellnet, has decided to stress low prices, and to list all the savings included in their "First for Families" special offer. Phone calls are then metonymically replaced by their costs. The firm Vodafone has decided to ignore mobile phones altogether, and concentrate instead on the social networks which spur the development of the telecom industry. The rationale of mobile phone advertisements thus appears to be based on various levels of metonymies, from physical telecom to economic and social networks.

\section{INDEX}

Mots-clés : métaphore, métonymie, publicité, réseau, téléphone

Keywords : advertisement, metaphor, metonymy, network, telephone 


\section{AUTEUR}

MARIE-HÉLĖNE FRIES-VERDEIL

Marie-Hélène Fries-Verdeil est maître de conférences à l'université de Grenoble 1, où elle enseigne l'anglais scientifique en DEUG de biologie. Ses centres d'intérêt en matière de recherche sont la publicité scientifique et les métaphores. Elle est co-auteur de Speaking Skills for Scientific English. Marie-Helene.Fries@ujf-grenoble.fr 